\title{
Detecting Commuting Patterns by Clustering Subtrajectories
}

Kevin Buchin

Maike Buchin

Joachim Gudmundsson

Maarten Löffler

Jun Luo

Technical Report UU-CS-2008-029

September 2008

Department of Information and Computing Sciences

Utrecht University, Utrecht, The Netherlands

www.cs.uu.nl 
ISSN: 0924-3275

Department of Information and Computing Sciences Utrecht University P.O. Box 80.089 3508 TB Utrecht The Netherlands 


\title{
Detecting Commuting Patterns by Clustering Subtrajectories*
}

\author{
K. Buchin ${ }^{\dagger} \quad$ M. Buchin ${ }^{\dagger} \quad$ J. Gudmundsson ${ }^{\ddagger} \quad$ M. Löffler ${ }^{\dagger} \quad$ J. Luo $^{\dagger}$
}

\begin{abstract}
In this paper we consider the problem of detecting commuting patterns in a trajectory. For this we search for similar subtrajectories. To measure spatial similarity we choose the Fréchet distance and the discrete Fréchet distance between subtrajectories, which are invariant under differences in speed. We give several approximation algorithms, and also show that the problem of finding the 'longest' subtrajectory cluster is as hard as MaxClique to compute and approximate.
\end{abstract}

\section{Introduction}

Technological advances of location-aware devices, surveillance systems and electronic transaction networks produce more and more opportunities to trace moving entities. Consequently, a variety of disciplines including geography, market research, data base research, animal behaviour research, surveillance, security and transport analysis shows an increasing interest in movement patterns of entities moving in various spaces over various times scales [19, 29, 44]. In the case of moving animals, movement patterns can be viewed as the spatio-temporal expression of behaviours, e.g. flocking sheep or the seasonal migration of birds. In a transportation context, a movement pattern could be a traffic jam or a commuting route.

In this paper we will focus on the problem of detecting commuting patterns in a trajectory. For this we search for similar subtrajectories. To measure spatial similarity we choose the Fréchet distance and the discrete Fréchet distance between subtrajectories. Both of these are invariant under differences in speed: for instance, in a transportation context, this allows to detect a commuting pattern even in the presence of different traffic conditions and varying means of transport. We will also consider how to detect a common movement pattern of a group of entities. That is, we want to find similar subtrajectories in a given set of trajectories. This problem can be handled using the same approach as for one entity. We will focus on the problem for one entity, but also discuss the changes for a group of entities. Figure 1 shows examples for the two different problem statements, i.e., similar subtrajectories of a single trajectory and of several trajectories.

More formally, the input is a moving point object, called entity, whose location is known at $n$ consecutive time-steps. Thus, the trajectory of an entity is a polygonal curve that can selfintersect. We assume that an entity moves between two consecutive time steps on a straight line. Given the trajectory $T$ of a moving entity in the plane, an integer $m>0$ and two positive real values $\ell$ and $d$, we define a commuting pattern as a set of $m$ subtrajectories of $T$, where the time intervals of two subtrajectories overlap in at most a point, the subtrajectories are within

*This research was initiated during the GADGET Workshop on Geometric Algorithms and Spatial Data Mining, funded by the Netherlands Organisation for Scientific Research (NWO) under BRICKS/FOCUS grant number 642.065.503. The research was further supported by NWO through the GADGET and GOGO projects, and by the Australian Government's Backing Australia's Ability initiative, in part through the Australian Research Council.

${ }^{\dagger}$ Department of Information and Computing Sciences, Utrecht University, the Netherlands. \{buchin,maike, ljroger, loffler\}@cs.uu.nl

$\ddagger$ NICTA, Sydney, Australia. joachim.gudmundsson@nicta.com.au 
(a)

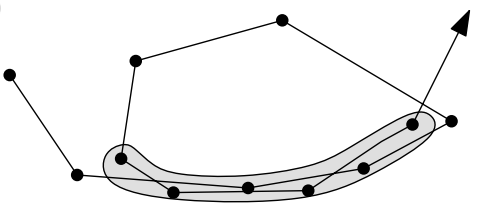

(b)

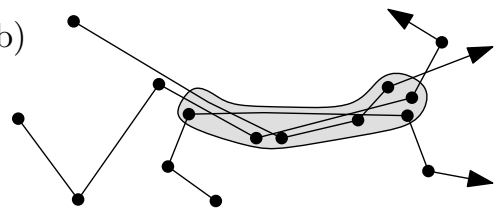

Figure 1: (a) The two subtrajectories within the shaded region form a subtrajectory cluster for $m=2$ if the length of the longest subtrajectory is longer than $\ell$ and the Fréchet distance between the two subtrajectories is at most $d$. (b) Illustrating a subtrajectory cluster for $m=3$ in the case when a set of trajectories is given as input.

distance $d$ from each other, and at least one subtrajectory has length $\ell$ between its first and last vertex. See Figure 1 for an example. As mentioned above, we will use the Fréchet distance and the discrete Fréchet distance as distance measures, which are natural distance measures for curves and polygonal curves.

Recently there has been considerable research in the area of analysing and modelling spatiotemporal data [25]. In the database community, research has mainly focused on indexing databases so that basic spatio-temporal queries concerning the data can be answered efficiently. Typical queries are spatio-temporal range queries, spatial or temporal nearest neighbours, see for example the work by Săltenis et al. [40] and Hadjieleftheriou et al. [30]. From a data mining perspective, Verhein and Chawla [42] used association rule mining to detect patterns in spatio-temporal sets. They defined a region to be a source, sink or a thoroughfare depending on the number of objects entering, exiting or passing through the region. Recently there have been several papers considering the problem of detecting flock, leadership, convergence and single file patterns [3, 7, 8, 9, 10, 24, 32].

Precursory to this work, Laube et al. $[34,35]$ proposed the REMO framework (RElative MOtion) which defines similar behaviour in groups of entities. They defined patterns such as 'flock', 'convergence', 'trend-setting' and 'leadership' based on similar movement properties such as speed, acceleration or movement direction; and they gave algorithms to compute them efficiently. They proposed an input model where a ray was drawn from the current position of each entity that corresponds to its direction. That is, the coordinates and direction of the entities are known at the initial time step, and their aim is to find or forecast a popular place (assuming the entities do not change their direction).

Vlachos et al. [43] state that in the area of spatio-temporal analysis it is an important task to detect commuting patterns of a single entity, or to find objects that moved in a similar way. Thus, an efficient clustering algorithm for trajectories, or subtrajectories, is essential for such data analysis tasks. Gaffney et al. [20, 21] proposed a model-based clustering algorithm for trajectories. In this algorithm, a set of trajectories is represented using a regression mixture model. Specifically, their algorithm is used to determine the cluster memberships and it only clusters whole trajectories. Lee et al. [38] argued that clustering trajectories as a whole could not detect similar portions of the trajectories. They instead suggested an approach that partitions a trajectory into a set of line segments and then group similar line segments. The obvious drawback is that only segments are clustered, while a commuting pattern might be a complicated path whose shape may not be captured by a single segment. Mamoulis et al. [36] used a different approach to detect periodic patterns. They assumed that the trajectories are given as a sequence of spatial regions, for example $A B C$ would denote that the entity started at region $A$ and then moved to region $C$ via region $B$. Using this model data mining tools such as association rule mining can be used.

Vlachos et al. [43] also looked at discovering similar trajectories of moving objects. They mainly focused on formalising a similarity function based on the longest common subsequence which they claim is very robust to noise. However, their approach matches the vertices along the trajectories which requires that the vertices along the trajectories are synchronised, or almost synchronised. 
Since it is not unusual that the coordinates are recorded with a frequency of five per second it has recently been argued $[13,26]$ that this is an unreasonable assumption since trajectories will have to be compressed (simplified) to allow for fast computations. In this paper we will make no such assumptions on the input data.

The paper is organised as follows. In the next section we introduce the Fréchet distance and formally define the problem considered in this paper. In Section 3 we prove that the longest subtrajectory cluster problem is as hard as MAXCLIQUE to approximate. We therefore consider approximation algorithms in Section 4.

\section{Preliminaries}

In this paper we will present algorithms that find clusters of subtrajectories of a given trajectory. The idea is to select a reference subtrajectory and then to find all subtrajectories that are close to this using the Fréchet distance. The proposed algorithm can be extended to handle subtrajectory clustering in the case when the input is a set of trajectories and the aim is to find the longest subtrajectory cluster where each subtrajectory in the cluster is a subtrajectory of an input trajectory and no two trajectories in the cluster belong to the same input trajectory.

As mentioned in earlier work [9, 24], specifying exactly which of the patterns should be reported is often a subject for discussion. In this paper we consider the problems of finding the longest cluster of a fixed size and the largest cluster of a fixed length. We say that a cluster $C$ of (sub)trajectories has length at least $\ell$ if there exists a (sub)trajectory in $C$ whose length between the first and last vertex is at least $\ell$.

Definition 1 Given a trajectory $T$ with $n$ vertices, a subtrajectory cluster $C_{T}(m, \ell, d)$ for $T$ of length $\ell$ consists of at least $m$ non-identical subtrajectories $T_{1}, \ldots, T_{m}$ of $T$ such that the time intervals for two subtrajectories overlap in at most a point, the distance between the subtrajectories is at most $d$, and at least one subtrajectory has length $\ell$.

Problem 1 (Subtrajectory Cluster - SC $(m, \ell, d)$ ) Given a trajectory $T$, the subtrajectory cluster problem $\mathrm{SC}(m, \ell, d)$ is the decision problem: does there exist a subtrajectory cluster with these parameters? We also define the related optimisation problems $\mathrm{SC}(\max , \ell, d), \mathrm{SC}(m, \max , d)$, and $\mathrm{SC}(m, \ell, \min )$, which keep two parameters fixed and aim to maximise or minimise the third.

A variant of this problem is to find a subtrajectory cluster in a given set of trajectories, as shown in Figure 1(b). This variant has two further subvariants: for each trajectory at most one subtrajectory is allowed, or several. By concatenating the given set of trajectories to one trajectory, any instance of this problem can be made into an instance of the subtrajectory cluster problem. For the subvariant where only one subtrajectory of each trajectory is allowed, this restriction has to be enforced, as well. This subvariant can be seen as a partial matching problem of curves. Partial matching problems for two curves using the Fréchet distance that have been considered are matching a curve to a subcurve of a different trajectory [4] and matching two curves where several subcurves may contribute to the matching [12].

In Definition 1 we omitted to define the distance metric. In previous papers several different distance functions and approaches have been used, for example the Longest Common Subsequence model [43], a combination of parallel distance, perpendicular distance and angle distance [38], the count of common subsequences of length two [2], the domain-dependent extraction of a single representative value for the whole series [33], the average Euclidean distances between paths [37]. An alternative to the Fréchet distance taking obstacles into account is the geodesic Fréchet distance $[15]$. 
The Fréchet distance is a distance measure for continuous shapes such as curves and surfaces, and is defined using reparameterisations of the shapes. Since it takes the continuity of the shapes into account it is generally regarded as being a more appropriate distance measure than the Hausdorff distance for curves $[5,6,41]$. For polygonal curves, the discrete Fréchet distance is a natural variant of the Fréchet distance. In this paper we will use the Fréchet distance [4] and discrete Fréchet distance [17]. For a set of $m>2$ curves there is a natural extension due to Dumitrescu and Rote [16]. We will denote the Fréchet distance between to curves $f$ and $g$ by $\delta_{F}(f, g)$, and the discrete Fréchet distance by $\delta_{d F}(f, g)$. For completeness, we include the formal definitions of these distance measures here.

Definition 2 Let $f: I=\left[l_{I}, r_{I}\right] \rightarrow \mathbb{R}^{c}$ and $g: J=\left[l_{J}, r_{J}\right] \rightarrow \mathbb{R}^{c}$ be two curves, and let $|\cdot|$ denote the Euclidean norm ${ }^{1}$ in $\mathbb{R}^{c}$. Then the Fréchet distance $\delta_{F}(f, g)$ is defined as

$$
\delta_{F}(f, g)=\inf _{\substack{\alpha:[0,1] \rightarrow I \\ \beta:[0,1] \rightarrow J}} \max _{t \in[0,1]}|f(\alpha(t))-g(\beta(t))|,
$$

where $\alpha$ and $\beta$ range over continuous and increasing functions with $\alpha(0)=l_{I}, \alpha(1)=r_{I}, \beta(0)=l_{J}$ and $\beta(1)=r_{J}$.

For a set of $m>2$ curves there is a natural extension due to Dumitrescu and Rote [16].

Definition 3 For a set of $m$ curves $\mathcal{F}=\left\{f_{1}, \ldots, f_{m}\right\}, f_{i}:\left[a_{i}, a_{i}^{\prime}\right] \rightarrow \mathbb{R}^{2}$ we define the Fréchet distance as

$$
\begin{aligned}
\delta_{F}(\mathcal{F})= & \inf _{\alpha_{1}:[0,1] \rightarrow\left[a_{1}, a_{1}^{\prime}\right] \quad} \max _{t \in[0,1] 1 \leq i, j \leq m}\left|f_{i}(\alpha(t))-f_{j}(\beta(t))\right|, \\
& \vdots \\
& \alpha_{m}:[0,1] \rightarrow\left[a_{m}, a_{m}^{\prime}\right]
\end{aligned}
$$

where $\alpha_{1}, \ldots, \alpha_{m}$ range over continuous and increasing functions with $\alpha_{i}(0)=a_{i}$ and $\alpha_{i}(1)=a_{i}^{\prime}$, $i=0, \ldots, m$.

In this paper we will also consider the discrete Fréchet distance, which is a variant of the Fréchet distance for polygonal curves. For the discrete Fréchet distance only distances between vertices are computed. It is defined using couplings of the vertices of the curves [17]. Consider two polygonal curves $P, Q$ in $\mathbb{R}^{c}$ given by the sequences of their vertices $\left\langle p_{1}, \ldots, p_{n}\right\rangle$ and $\left\langle q_{1}, \ldots, q_{m}\right\rangle$, respectively. A coupling $C$ of the vertices of $P, Q$ is a sequence of pairs of vertices $C=\left\langle C_{1}, \ldots, C_{k}\right\rangle$ with $C_{r}=\left(p_{i}, q_{j}\right)$ for all $r=1, \ldots, k$ and some $i \in\{1, \ldots, n\}, j \in\{1, \ldots, m\}$, fulfilling

- $C_{1}=\left(p_{1}, q_{1}\right)$ and $C_{k}=\left(p_{n}, q_{m}\right)$

- $C_{r}=\left(p_{i}, q_{j}\right) \Rightarrow C_{r+1} \in\left\{\left(p_{i+1}, q_{j}\right),\left(p_{i}, q_{j+1}\right),\left(p_{i+1}, q_{j+1}\right)\right\}$ for $r=1, \ldots, k-1$.

Definition 4 Let $P, Q$ be two polygonal curves in $\mathbb{R}^{c}$. Let $|\cdot|$ denote an underlying norm on $\mathbb{R}^{c}$. Then the discrete Fréchet distance $\delta_{d F}(f, g)$ is defined as

$$
\delta_{d F}(f, g)=\min _{\text {coupling }} \max _{\left(p_{i}, q_{j}\right) \in C}\left|p_{i}-q_{j}\right|,
$$

where $C$ ranges over all couplings of the vertices of $f$ and $g$.

The study of the Fréchet distance from a computational point of view was initiated by Alt and Godau [4]. They showed that the Fréchet distance between two curves with $m$ and $n$ segments respectively can be computed in $O(m n \log m n)$ time, and the associated decision question (is

\footnotetext{
${ }^{1}$ Other norms are possible as well, see [4].
} 
the Fréchet distance more than $d$ ?) can be answered in $O(m n)$ time assuming the model of computation can do square roots in constant time. In the following we will assume that the model of computation can do this for all results concerning the Fréchet distance. This is not necessary for the discrete Fréchet distance. The study of the discrete Fréchet distance was started by Eiter and Mannila [17]. They gave a simple dynamic programming algorithm for computing the discrete Fréchet distance in $O(m n)$ time. Furthermore, they showed that $\delta_{F}(f, g) \leq \delta_{d F}(f, g) \leq$ $\delta_{F}(f, g)+L / 2$, where $L$ is the length of the longest segment of $f$ or $g$. This implies that under sufficient sampling, the discrete Fréchet distance is an arbitrary good approximation of the Fréchet distance. For the decision question of the Fréchet distance and of the discrete Fréchet distance a $\Omega((m+n) \log (m+n))$ lower bound holds in the algebraic computation tree model allowing arithmetic operations and tests [11]. Sometimes we will use the term continuous Fréchet distance to contrast the Fréchet distance to the discrete Fréchet distance.

In this paper we will focus on the optimisation version of Problem 1 where the length $\ell$ is maximised, i.e., the longest subtrajectory cluster $\mathrm{SC}(m, \max , d)$ for fixed parameters $m$ and $d$. We will prove in the next section that the general decision problem $\mathrm{SC}(m, \ell, d)$ is NP-complete, so the optimisation variants are also NP-hard. Therefore, we will turn our attention to approximation algorithms. We speak of a size approximation when we approximate $m$, a length approximation when we approximate $\ell$, and a distance approximation when we approximate $d$. For instance, assume an optimal solution of $\mathrm{SC}(m, \max , d)$ has length $\ell^{*}$. A $c$-distance approximation algorithm for this problem would return a cluster $C_{T}\left(m, \ell^{*}, c d\right)$ of length at least $\ell^{*}$ and with $m$ subtrajectories of $T$ within Fréchet distance $c \cdot d$ from each other. We will also briefly discuss the case where the size of the cluster is maximised, i.e., the problem $\mathrm{SC}(\max , \ell, d)$ for fixed $\ell, d$.

In the following, for an instance of the SC problem we will always use $n$ to denote the number of vertices in the given trajectory, $\ell$ for the length of the longest subtrajectory cluster, $m$ for the size of the cluster, and $d$ for the distance between two curves in the cluster. If the input is a set of trajectories, we will use $k$ for the number of trajectories and $n$ for the maximum length of a single trajectory.

\section{$3 \quad$ Hardness Results}

In this section we give several hardness results. We will prove that the general decision problem $\mathrm{SC}(m, \ell, d)$ is NP-complete, so the optimisation variants are also NP-hard and we will have to turn our attention towards approximation algorithms. We will also prove some hardness results for several approximation problems. First we prove that any distance approximation of SC is 3sum-hard. Then we show that the general problem is NP-complete, and prove that the problems of finding a subtrajectory cluster containing a maximum number of subtrajectories or having maximum length are NP-hard to approximate within a factor of $n^{1-\varepsilon}$ for any positive constant $\varepsilon$, even if we allow a factor $2-\phi$ distance approximation. This also motivates why we study 2-distance approximation algorithms in the following sections.

The results in this section follow from similar results in [24].

\subsection{3 -SUM-Hardness}

Lemma 1 Finding any distance approximation of the $\mathrm{SC}(m, \max , d)$ problem is 3sum-hard.

Proof. To prove this, we make a reduction from the problem PoINT-ON-3-LINEs to a special case of our clustering problem.

In the POINT-ON-3-Lines problem, you are given a set of lines and asked the question whether there is any point that is on three of the lines simultaneously. This problem was proven to be 
3Sum-hard by Gajentaan and Overmars [22]: this means that it is at least as hard as 3sum for which no subquadratic time algorithm has been found yet, which is an indication of the inherent hardness of the problems. For a weak model of computation a lower bound of $\Omega\left(n^{2}\right)$ for those problems exists [18].

Consider a set $S=\left\{s_{1}, \ldots, s_{n}\right\}$ of $N$ lines in the plane. Compute in $O(n \log n)$ time a bounding box $B$ such that all intersections between lines in $S$ occur within $B$. Remove the parts of the lines outside $B$ such that we obtain a set $S^{\prime}=\left\{s_{1}, \ldots, s_{n}\right\}$ of $N$ segments, and connect the endpoints outside $B$ such that we obtain one trajectory $T$. The segments can be connected by polygonal paths outside $B$ in such a way that no three lines intersect in a point outside $B$.

Assume we have an approximation algorithm $A$ that computes, for a given trajectory and parameters $m$ and $d$, A set of $m$ subtrajectories of length $\ell$ that are all within distance $c \cdot d$ from each other, where $\ell$ is the optimal value for these $m$ and $d$. Then we can run this algorithm on $T$ with parameters $m=3$ and $d=0$. If we obtain any solution, then there must be three lines in $S$ that intersects in a common point, otherwise no such point exists. The observation follows.

\section{$3.2 \quad$ NP-Hardness}

Theorem 1 The decision problem $\mathrm{SC}(m, \ell, d)$ is NP-complete.

Proof. The problem is obviously in NP since a candidate subtrajectory cluster can be verified in polynomial time by computing the Fréchet distance between every pair of subtrajectories in the candidate cluster.

Next, we prove NP-hardness by a reduction from MaxCLIQue [23]. In this problem, you are given a graph $G=(V, E)$ with $n$ vertices $v_{1}, \ldots, v_{n}$, and want to determine whether a clique of size $m$ exists: a complete subgraph with $m$ vertices.

We construct an instance of the SC problem as follows, see Fig. 2. We choose the distance $d=1$; the instance can be scaled to realise any value of $d$. Every vertex $v$ in $V$ is represented by a subtrajectory $T_{v}$ containing $3 n+1$ points. We define the locations of all subtrajectories at all steps as follows. The first point of a subtrajectory is at the origin of the plane. For vertex $v_{j}$ and $0<i \leq n$, the trajectory $T_{v_{j}}$ at step $3 i$ is at $(3 i, 0)$ and at steps $3 i-1$ and $3 i-2$ is at

- $(3 i-1,1)$ and $(3 i-2,1)$ if $i=j$,

- $(3 i-1,0)$ and $(3 i-2,0)$ if $i \neq j$ and $\left(v_{i}, v_{j}\right) \in E$, and

- $(3 i-1,-1)$ and $(3 i-2,-1)$ if $i \neq j$ and $\left(v_{i}, v_{j}\right) \notin E$.

We connect all consecutive subtrajectories to obtain the trajectory $T$. Note that two subtrajectories can easily be connected by a path containing two edges, in such a way that no two subpaths connecting two subtrajectories are close to each other.

We observe that in the time interval between $3 i-3$ and $3 i$, any set of subtrajectories containing the $i$-th subtrajectory and the subtrajectories that correspond to the vertices which are connected to $v_{i}$ in $G$ will have Fréchet distance at most $d$ while any set of subtrajectories obtaining the $i$-th subtrajectory and the subtrajectories that correspond to the vertices who are connected to $v_{i}$ in $G$ will have Fréchet distance greater than $d$. We observe that two subtrajectories $T_{v_{i}}$ and $T_{v_{j}}$ have Fréchet distance at most $d$ if the vertices $v_{i}$ and $v_{j}$ are connected by an edge. Hence, the question whether a clique of size $m$ exists in $G$ can be answered by answering the question whether a subtrajectory cluster of size $m$ exists for the duration of all $\ell=3 n+1$ time steps. This proves the NP-completeness of the decision version of the maximum subset SC problem. 

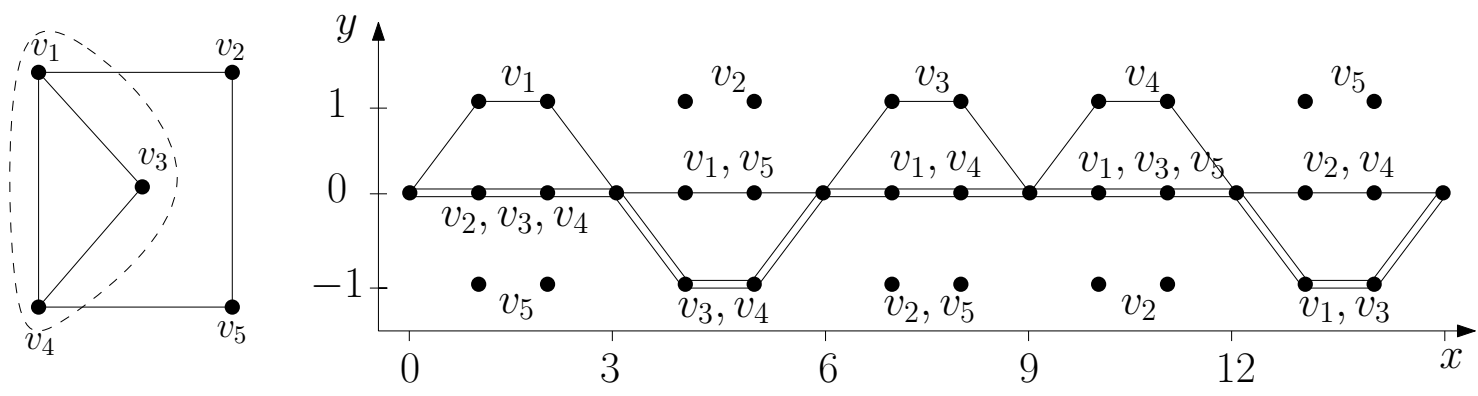

Figure 2: Illustrating the reduction in the proof of Theorem 1, in particular showing the subtrajectories corresponding to the 3 -Clique $v_{1}, v_{3}, v_{4}$.

The proof of Theorem 1 holds for any Fréchet distance in the interval $[1,2)$. Hence, even if we allow an approximate distance with a factor less than 2, the problem is still NP-hard.

Corollary 1 The problem of computing a $(2-\phi)$-distance approximation of $\mathrm{SC}(\max , \ell, d)$, for any $0<\phi \leq 1$, is $N P$-hard.

The above result can easily be strengthened by noting that an $\alpha$-approximation of the size of $\mathrm{SC}(\max , \ell, d)$ also corresponds to an $\alpha$-approximation of MAXCLIQUE, that is, we cannot hope to find a $\mathrm{SC}$ of approximately the maximum size efficiently. We restate from [31]:

Fact 1 (Håstad 1999)

For any constant $\varepsilon>0$, MAXCLIQUE cannot be approximated in polynomial time within a factor of $n^{1 / 2-\varepsilon}$ unless $P=N P$, and not within a factor of $n^{1-\varepsilon}$ unless $N P=Z P P$.

Corollary 2 The problem of computing a $n^{1 / 2-\varepsilon}$-size, $(2-\phi)$-distance approximation of the $\mathrm{SC}(\max , \ell, d)$ problem, for any $\phi, \varepsilon>0$, is NP-hard.

However, the problem we will focus on in this paper is to find the longest subtrajectory cluster. A similar result as in the above corollary can be obtained for this problem in the following way. We build the same construction as in the proof of Theorem 1 (in the appendix) for an instance of MAxClique with $N$ vertices. Note that the number of trajectories in such a construction is $N$ and the length of the trajectories is also $N$. Let $0<\varepsilon<1$ be a positive real constant and set $\tau=N^{1 / \varepsilon}$. Connect $\tau^{1-\varepsilon}$ copies of the constructions which gives an instance with $N$ trajectories each of length $\tau$. This instance contains a subtrajectory cluster of length $\tau$ and size $N$ if and only if each copy contains a subtrajectory cluster of size and length $N$ and this exists if and only if a MaxClique of size $N$ exists. However this is NP-complete, and hence, computing a longest subtrajectory cluster within an approximation factor of $\tau / N=\tau^{1-\varepsilon}$ is NP-hard. We have:

Corollary 3 The problem of computing a $\tau^{1-\varepsilon}$-length, $(2-\phi)$-distance approximation of the $\mathrm{SC}(m, \max , d)$ problem, for any $\phi, \varepsilon>0$, is NP-hard.

\section{A 2-Distance Approximation using the Fréchet Distance}

First we briefly outline the free space diagram of two polygonal curves $f$ and $g$, which is a geometric data structure introduced by Alt and Godau [4] for computing the Fréchet distance. Let 
$f$ be a polygonal curve with $n$ vertices $p_{1}, \ldots, p_{n}$. We use $\phi_{f}$ to denote the following natural parameterisation of $f$. The map $\phi_{f}:[1, n] \rightarrow \mathbb{R}^{c}$ maps $i \in\{1, \ldots, n\}$ to $p_{i}$ and interpolates linearly in between vertices, where $c$ is any dimension. The free space diagram of two polygonal curves $f$ and $g$, with $n$ and $m$ vertices, respectively, is the set

$$
F_{d}(f, g)=\left\{(s, t) \in[1, n] \times[1, m]:\left|\phi_{f}(s), \phi_{g}(t)\right| \leq d\right\}
$$

which describes all tuples $(s, t)$ such that the points $\phi_{f}(s)$ and $\phi_{g}(t)$ have Euclidean distance at most $d$. See Figure 3 for an example of a free space diagram. The complexity of the free space diagram is $O(n m)$ and it can be constructed in time $O(n m)$. Alt and Godau [4] showed that the Fréchet distance between $f$ and $g$ is less than $d$ if and only if there exists a monotone path in the free space diagram $F_{d}(f, g)$ from $(0,0)$ to $(n, m)$.
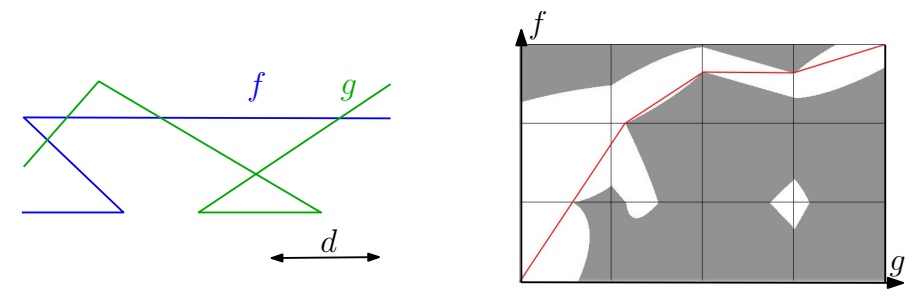

Figure 3: Polygonal curves $f$ and $g$, distance value $d$, and a monotone path in the free space diagram.

For the discrete Fréchet distance the free space diagram consists of the $n m$ grid points. Each grid point $(x, y)$ is labelled free or not depending on whether $\left|\phi_{f}(x)-\phi_{g}(y)\right|$ is less than or equal to the distance $d$ or not. A monotone path is a sequence of free grid points from $(0,0)$ to $(n, m)$ where a grid point $(x, y)$ is followed either by $(x+1, y),(x, y+1)$ or $(x+1, y+1)$ [17]. Analogous to the continuous Fréchet distance, it holds that the discrete Fréchet distance is less than $d$ if and only if there is a monotone path in the free space diagram.

Consider a set of $m$ polygonal curves $\mathcal{F}=\left\{f_{1}, \ldots, f_{m}\right\}$, where each curve $f_{i}$ has $n_{i}$ segments $(i=1, \ldots, m)$. The Fréchet distance of the set $\mathcal{F}$ can be computed in time $O\left(\left(n_{1} \cdot \ldots \cdot n_{m}\right) \log \left(n_{1}\right.\right.$. $\left.\left.\ldots \cdot n_{m}\right)\right)$. Even for small values of $m$ this is deemed impractical. However, Dumitrescu and Rote [16] showed that a 2-approximation of the distance can be obtained by computing all the pairwise Fréchet distances and outputting $\min _{1 \leq i \leq m} \max _{1 \leq j<k \leq m}\left(\delta_{F}\left(f_{i}, f_{j}\right)+\delta_{F}\left(f_{i}, f_{k}\right)\right)$, which only requires time $O\left(\sum_{1 \leq i<j \leq m} n_{i} n_{j} \log n_{i} n_{j}\right)$. For the discrete Fréchet distance the same approximation holds which can be computed in time $O\left(\sum_{1 \leq i<j \leq m} n_{i} n_{j}\right)$.

Cluster Curves in the Free Space. In our application, we have just one polygonal curve: this input trajectory $T$. Now consider the free space diagram $F_{d}(T, T)$ of two copies of $T$, and distance value $d$. Let $s, t \in[1, n]$ be two points in time for the trajectory $T$, and let $l_{s}$ and $l_{t}$ be the two vertical lines in $F_{d}(T, T)$ corresponding to them. For the discrete Fréchet distance, we assume that $s, t \in\{1, \ldots, n\}$, i.e., $l_{s}$ and $l_{t}$ fall on vertices in $F_{d}(T, T)$, see Figure 4 . For simplicity we assume that the $x$ - and $y$-axis of the diagram goes from 1 to $n$, i.e., from $v_{1}$ to $v_{n}$.

Definition 5 We say that there are $m$ cluster curves between $l_{s}$ and $l_{t}$ in $F_{d}(T, T)$ if and only if there are $m$ non-identical curves starting at $l_{s}$ and ending at $l_{t}$ such that:

- each curve is monotonically increasing in both coordinates from $l_{s}$ to $l_{t}$, and

- the y-coordinates of two curves overlap in at most a point. 

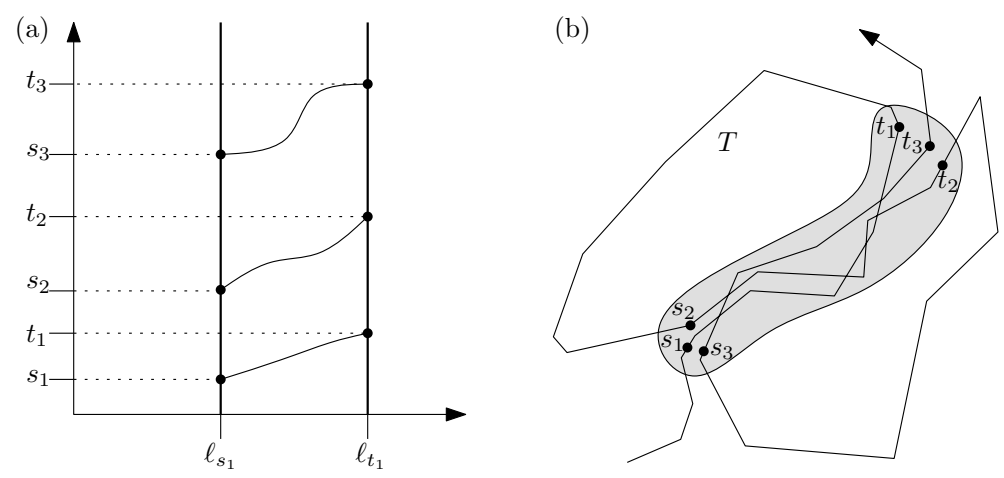

Figure 4: (a) There exists a trajectory cluster with three subtrajectories between $s$ and $t$. A possible trajectory corresponding to the cluster curves is illustrated in (b). Note that the subtrajectory $s, t$ is one of the subtrajectories.

Figure 4 shows an example, and the corresponding cluster of subtrajectories of $T$.

We will make use of the two important implications, which are necessary for the algorithm in Section 4. For this, we use the following lemma of Alt and Godau [4].

Lemma 2 (Alt and Godau [4])

Two polygonal curves $f$ and $g$ in the plane, with $n$ and $m$ vertices respectively, have Fréchet distance $\delta_{F}(f, g) \leq d$, if and only if there exists a curve in the corresponding free space diagram $F_{d}(f, g)$ from $(0,0)$ to $(n, m)$ that is monotone in both coordinates.

We now state the lemma and present its proof.

Lemma 3 Let $T$ be trajectory and $T^{\prime}$ the subtrajectory of $T$ that starts at $s$ and ends at $t$, where $t-s=\ell$. Then the following holds:

- If there exist $m$ cluster curves within the free space diagram $F_{d}(T, T)$ between $l_{s}$ and $l_{t}$, then there exists a subtrajectory cluster $C_{T}(m, \ell, 2 d)$ containing $T^{\prime}$.

- If there exists a subtrajectory cluster $C_{T}(m, \ell, d)$ containing $T^{\prime}$, then there exists a set of $m$ cluster curves within the free space diagram $F_{d}(T, T)$ between $l_{s}$ and $l_{t}$.

Proof. Let $C=\left\{c_{1}, \ldots, c_{m}\right\}$ be the set of $m$ cluster curves within the free space diagram $F_{d}(T, T)$ from $s$ to $t$. According to Lemma 2 this implies that the pairwise Fréchet distance between every pair of subtrajectories corresponding to the curves is at most $d$. Since this holds for every pair of curves it follows from the result by Dumitrescu and Rote [16] that the set of trajectories has Fréchet distance at most $2 d$. Finally, since the $y$-coordinates of two curves in $C$ overlap in at most a point, the time intervals for two subtrajectories also overlap in at most point. This completes the proof of the first part.

Let $C$ be the solution to $\mathrm{SC}(m, \ell, d)$ containing $T^{\prime}$, i.e., $C=\left\{f_{1}, \ldots, f_{m}\right\}$ is a set of subtrajectories of $T$ and the Fréchet distance of $F$ is at most $d$. This implies that also all pairwise Fréchet distances in $C$ are at most $d$. W.l.o.g. let $T^{\prime}=f_{1}$ the subtrajectory from $t_{a}$ to $t_{b}$. From Lemma 2 it follows that for every trajectory in $C$ there exists a cluster curve within the free space diagram $F_{d}(T, T)$ from the $x$-coordinate $s$ to the $x$-coordinate $t$ that is monotone in both coordinates. Finally, since the time intervals for two subtrajectories overlap in at most a point, their corresponding cluster curves within $F_{d}(T, T)$ also overlap in at most a point. This completes the proof. 
Note that the above lemma also implies that if there is no subtrajectory cluster $C_{T}(m, \ell, 2 d)$ containing $T^{\prime}$ then there do not exist $m$ cluster curves between $t_{a}$ and $t_{b}$.

The simplest variant of the algorithm is using the discrete Fréchet distance. We first give and analyse this variant in Section 4.1 and then extend it to the continuous Fréchet distance in Section 4.2 .

\subsection{Discrete Fréchet Distance}

In this section we describe and analyse the algorithm for the discrete Fréchet distance.

\subsubsection{Algorithm}

Recall that as input we are given a trajectory $T$ with $n$ vertices, an integer $m$ and a positive real value $d$. Consider the free space diagram $F=F_{d}(T, T)$ of $T$. For the discrete Fréchet distance, this consists only of the at most $n^{2}$ grid points which lie in free space, i.e., where the distance between the corresponding two vertices is less than or equal to $d$. We sweep two vertical lines in $F: l_{s}$ and $l_{t}$, with $x$-coordinates $s$ and $t$, which represent the start point and the end point of the reference trajectory, respectively. Initially $l_{s}$ and $l_{t}$ are at the leftmost position in $F$. We will sweep $l_{s}$ and $l_{t}$ in discrete steps along the $x$-coordinates $\{1, \ldots, n\}$.

The algorithm proceeds as follows. Move $l_{t}$ to the right to the first position for which there are less than $m$ cluster curves between $l_{s}$ and $l_{t}$. Next move $l_{s}$ to the right until $l_{s}$ equals $l_{t}$ or there are at least $m$ cluster curves between $l_{s}$ and $l_{t}$. Then move $l_{t}$ again, etc. This continues until $l_{s}$ reaches the rightmost position in $F$. During the sweep we maintain the longest length of a reference trajectory for which there exist at least $m$ cluster curves between its endpoints. It is easily seen that the two sweep lines of $l_{s}$ and $l_{t}$ move monotonically from left to right over $n$ event points. This implies that only $O(n)$ possible pairs of start and endpoints are considered.

What remains to be done is to build and update a data structure that allows us to check if there are $m$ monotone curves between $l_{s}$ and $l_{t}$ that overlap in at most a point along the $y$-axis. For this, we will store the free space between $l_{s}$ and $l_{t}$ as directed, labelled graph on the vertices in the free space. When updating $l_{t}$, we add a new column of the free space. When updating $l_{s}$, we delete the leftmost column. For checking whether $m$ curves exist between $l_{s}$ and $l_{t}$, we will search this graph. We will see that this data structure can be maintained in $O(n)$ time per step and using $O(\ell n)$ space where $\ell$ denotes the maximum number of vertices of a reference trajectory occurring in a cluster of $m$ curves.

Data structure. We store the free space between $l_{s}$ and $l_{t}$ as a directed, labelled graph on the vertices in the free space (see Figure 5). We store a directed edge from $p$ to $p^{\prime}$ if $p, p^{\prime}$ lie in the free space of the same cell and $p$ is to the left or above $p^{\prime}$. We label each edge by the smallest $x$-coordinate reachable by a path along this edge. This graph can be efficiently computed column-by-column from left to right, and bottom to top within a column. The directed edges encode the free space. The edge labels are easy to compute: An edge pointing to a vertex without outgoing edges is labelled with the $x$-coordinate of that vertex. An edge pointing to a vertex $p$ with outgoing edges is labelled by the smallest label of the outgoing edges of $p$. Since a vertex has outdegree at most three, an edge label can be computed in constant time.

Update. We can update this graph in $O(n)$ time per update: When $l_{t}$ is moved one position to the right, we need to compute the new column of the free space which has complexity $O(n)$. When $l_{s}$ is moved one position to the right, we simply delete the currently leftmost column of the free space. 
Query. Using our data structure, we can determine whether there are $m$ cluster curves between $l_{s}$ and $l_{t}$. We do this by greedily searching for monotone paths from $l_{t}$ to $l_{s}$. Let $i$ be the $x$ coordinate of $l_{s}$. We start at the topmost vertex on $l_{t}$ which has an outgoing edge labelled less or equal than $i$. In each vertex we follow the topmost edge which again has a label less or equal than $i$. This ends on a vertex $(i, j)$ on $l_{s}$. We continue with the topmost vertex on $l_{t}$ at height at most $j$ which as before has an outgoing edge labelled less or equal than $i$. We stop when we have found $m$ curves, or no more vertices on $l_{t}$ with an outgoing edge labelled less or equal than $i$ exist. When we find a horizontal path in the free space, i.e., a path from $\left(i^{\prime}, j\right)$ on $l_{t}$ to $(i, j)$ on $l_{s}$, we continue with the topmost vertex on $l_{t}$ at height at most $j-1$ (else we would continue finding the same horizontal path). Note that the edge labels prevent us from going into dead ends in the graph.

Since we need to ensure that the reference subtrajectory is part of the output cluster, must take care that the vertical span $[s, t]$ is not used in any of the curves. During the query, if the $y$ coordinate of current vertex gets smaller than $t$, then we don't increase the number of cluster curves and we skip down to $s$ and continue searching for cluster curves from the vertex $(t, s)$.

This query takes $O(n+m \ell)$ time, because the $m$ curves will in total consist of at most $n$ edges directed downward and $m \ell$ edges directed to the left (where we count diagonal edges as either down or left directed). There may not be more than $n$ edges directed downward since the $m$ curves cannot overlap in more than a point.
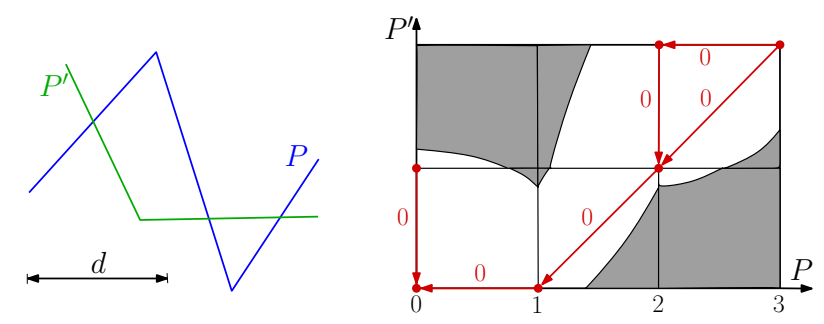

Figure 5: Illustrating the associated data structure.

\subsubsection{Analysis}

The description of the algorithm immediately gives the following lemma. Note that in practice, $m \ell$ should be of order $n$. Note that in practice, $m \ell$ should be of order $n$, i.e., the length $\ell$ is roughly the total length of $T$ divided by the number of curves in the cluster, that is $n / m$. In theory, other situations can occur, see Section 7.

Lemma 4 The data structure of the algorithm of size $O(n \ell)$ can be maintained in $O(n)$ time per update such that number-of-cluster-curves queries can be answered in $O(n+m \ell)$ time.

In total we have the following theorem.

Theorem 2 A 2-distance approximation of the $\mathrm{SC}(m, \max , d)$ problem can be computed in $O\left(n^{2}+\right.$ $n m \ell)$ time and $O(n \ell)$ space using the discrete Fréchet distance where $n$ denotes the number of vertices of the trajectory and $\ell$ denotes the maximum number of vertices occurring on a reference trajectory in a cluster of $m$ curves.

Proof. We need to prove both the complexity of the algorithm and its correctness. We start with the complexity. 
Since the two sweep lines, $l_{s}$ and $l_{t}$, move monotonically from left to right there will be $2 n$ events in total. At each event we perform an update and a query. According to Lemma 4 each update and query can be handled in $O(n+m \ell)$ time using $O(n \ell)$ space which implies that the total time and space complexity is $O\left(n^{2}+n m \ell\right)$ and $O(n \ell)$, respectively. Note that to reduce the space complexity of the algorithm, we do not precompute the free space diagram which has $O\left(n^{2}\right)$ size. Instead we only maintain the part of the free space which we are currently sweeping.

It remains to prove the correctness of the algorithm. Consider an optimal subtrajectory cluster $C$ containing $m$ subtrajectories and with Fréchet distance at most $d$. Let $\ell^{*}$ be the length of $C$, i.e., there exists a subtrajectory $\tau$ in $C$ such that the distance along $\tau$ between the first vertex $\left(v_{i}\right)$ to the last vertex $\left(v_{j}\right)$ on $\tau$ is $\ell^{*}$.

Consider the process of the algorithm, and consider the point during the sweep when $l_{s}$ reaches $v_{i}$. If $l_{t}$ has not passed $v_{j}$ then we know that there are at least $m$ cluster curves between $l_{s}$ and $l_{t}$ thus $l_{t}$ will be incremented. Note that this will continue until $l_{t}$ pass $v_{j}$, thus the algorithm will test $l_{s}=i$ and $l_{t}=j$ and report a subtrajectory cluster of length $\ell^{*}$. In the case when $l_{t}$ already passed $v_{j}$ then there must have been an integer $i^{\prime}$ such that $l_{s}=i^{\prime}$ and $l_{t}=j$ and $l_{t}$ was incremented. Since $i^{\prime}<i$ the length of this interval must have been greater than $\ell^{*}$. Furthermore, since $l_{t}$ was incremented the number of cluster curves must have been at least $m$. So a subtrajectory cluster starting at $v_{i^{\prime}}$ and ending at $v_{j}$ must have been reported. Consequently the subtrajectory cluster that is returned by the algorithm must have length at least $\ell^{*}$ in both cases. This proves the correctness of the algorithm.

\subsection{Fréchet Distance}

In this section we extend the algorithm to the continuous Fréchet distance. We first consider the case where subtrajectories in a cluster start and end at vertices and then further extend to arbitrary subtrajectories. Note that in our application it is realistic to assume that the subtrajectories in a cluster start and end at a vertex since a commuting route most often is between two places where the entity will stop and spend some time (i.e., work and home), hence generating data points.

\subsubsection{All Subtrajectories Start and End at Vertices}

We first consider the continuous Fréchet distance with the restriction that all subtrajectories start and end at vertices. In $F_{d}(T, T)$ each cell corresponds to two line segments of $T$ and the free space in one cell is the intersection of the cell with an ellipse, possibly degenerated to the space between two parallel lines [4]. There are at most eight intersection points of the boundary of the cell with free space. We call these intersection points critical points, see Figure 6 . For each critical point, we put a vertex on it. Then the grid of cells becomes a rectilinear graph $R=(V, E)$ with $|V|=O\left(n^{2}\right),|E|=O\left(n^{2}\right)$. Furthermore, we propagate all critical points in the free space of the corresponding row or column, respectively. That is, we propagate critical points on vertical cell boundaries horizontally, and critical points on horizontal cell boundaries vertically. Figure 6 shows an example of horizontal propagation. In particular, the figure shows an example where a monotone polygonal path is only possible with propagated critical points as vertices (e.g. the one shown by a solid red line).

In the following, we use $R$ to denote the set of propagated critical points and vertices of the free space. Using the propagated critical points and convexity of the free space in one cell we get, the following observation holds:

Observation 1 Let $v, w$ be two vertices of $R$. If there exists a monotonically increasing curve $P$ from $v$ to $w$ in the free space, then there exists a monotonically increasing polygonal path $P^{\prime}$ from $v$ to $w$ in the free space such that vertices of $P^{\prime}$ are vertices of $R$. 


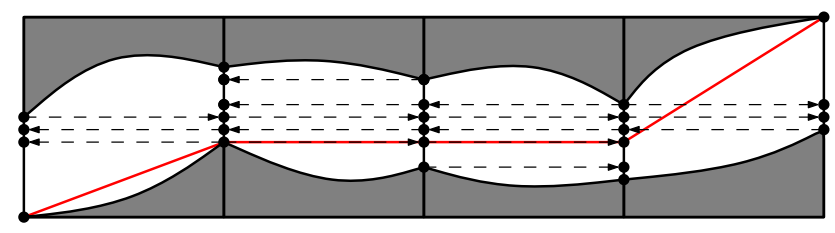

Figure 6: Adding and propagating critical points in the free space.

By Observation 1 it suffices to compute monotonically increasing polygonal paths using vertices of $R$. We can extend the data structure and algorithm for the discrete Fréchet distance to work with vertices of $R$ instead of only vertices of the free space. The size of $R$ is $O\left(n^{3}\right)$ since there are a linear number of critical points in each cell. However, in our algorithm we only store and update the part of the free space between the sweep lines $l_{s}$ and $l_{t}$. Furthermore, we will see next that we only need to explicitly compute the critical points on vertical cell boundaries. With this, we will get a space complexity of $O\left(n \ell^{2}\right)$ where $\ell$ is the length of a longest subtrajectory cluster.

For the query we are only interested in monotone paths between critical points on $l_{s}$ and $l_{t}$, i.e., only between critical points on vertical cell boundaries. Therefore we do not need to explicitly compute critical points on horizontal cell boundaries. Instead we can do the following. For all but the bottom-most point on a vertical cell boundary, it suffices to have at most a downward and a leftward directed edge. Because of the propagation the diagonal downward edge can be replaced by these two. The bottom-most point on a vertical cell boundary receives at most a leftward and a diagonal downward directed edge. The diagonal downward edge is labelled with two values, one is as before the left-most column index reachable by this edge. The other value stores which lower cells of this free space column are reachable from the point. Note that "reachable" for both edge labels refers to reachability in the graph encoding the free space, i.e., the direction is opposite to the direction of reachability in the free space.

More explicitly, consider the bottom-most point on the vertical cell boundary of the vertical line at column index $i+1$ in the $j$ th row. From this point, possibly the free space on vertical cell boundaries at column index $i$ in rows $j-1$ to $k$ for some $k \leq j-1$ are reachable. Whether the free space at column index $i$ in the $j$ th row is reachable, is stored in the possible leftward edge (i.e., there is an edge exactly if it is reachable). As second value for the downward edge, we store this value $k$ or we store $j$ if no lower cells are reachable. Furthermore, we store for each column index between $l_{s}$ and $l_{t}$ an array of $n$ pointers to the topmost points in free space intervals in all rows. Thus, when querying from a bottom-most point on a vertical cell boundary, we can jump to all lower free space cells that it can reach. In this way, we do not need to store any critical points on horizontal cell boundaries. We now show that these edge labels can be computed efficiently, that is in linear time per column, during the update of $l_{t}$.

Computing the Vertically Propagated Points Implicitly. Consider the column of the free space that is being added. From the cell in row $j$ we can reach a lower cell if the horizontal cell boundaries in between have a non-empty intersection. Formulated differently, for the horizontal cell boundaries in between the maximum left boundary is smaller or equal the minimum right boundary. We can test this condition for all columns using two doubly linked lists of length at most $n$, one for the left boundaries and the other for the right boundaries. See Figure 7 for an illustration. We iteratively add the horizontal cell boundaries from top to bottom, updating these lists in each step. The list entries contain three values: (1) the row index, (2) the position of the left or right boundary, respectively, at that row index, and (3) the furthest left column index reachable from this or a reachable upper cell of this column. For the right boundary, we are interested in small boundary positions, whereas for the left boundary we are interested in large boundary positions. We now describe the process for the right boundary list, for the left boundary 
list it is analogous.

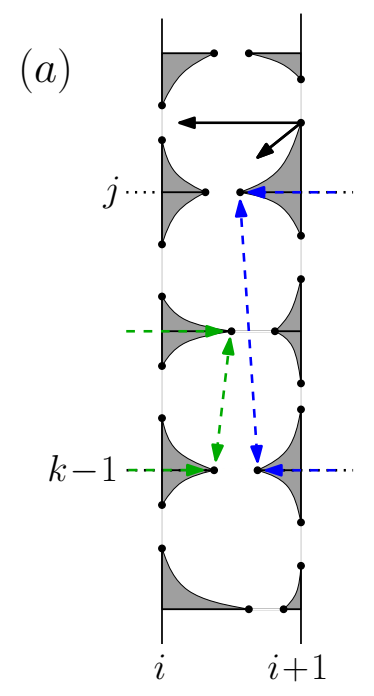

(b)

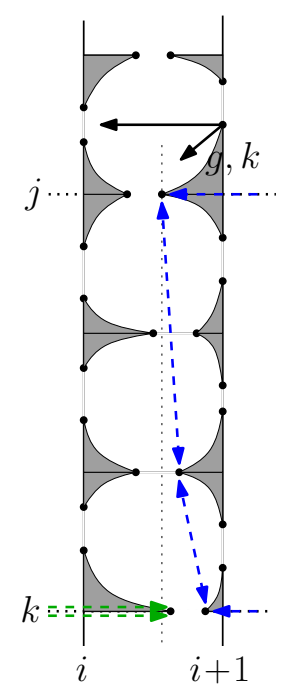

Figure 7: Updating $l_{t}$ for the continuous Fréchet distance. The doubly linked list are shown - by dashed arrows - (a) before and (b) after inserting the horizontal cell boundary at row index $k$.

The first list entry for the right boundary list stores the currently smallest boundary position at some row index $l$ (in Figure $7, l=j$ ). The next list entry stores the next smallest boundary position at a column index smaller than $l$, and so on. To add a new horizontal cell boundary, we first compute this cell boundary. If it is non-empty, we compare its position with the entries of the list, starting at the last entry. We delete all entries which are larger and store it at the first position where it is not smaller than the previous entry. During this process, we also compute the third list entry, i.e., the furthest reachable left column index. First we set this to the column index reachable in this row. Whenever a list entry is deleted (because its position was larger) we take the minimum of the current column index and the one stored by the entry which is being deleted. Finally, we also take the minimum with the column index stored by the last list entry not deleted (if this exists). The column indices stored in the lists will therefore be non-increasing. For one such step we may need to do a linear number of comparisons and deletions, however in total each entry is inserted and deleted exactly once in the process. After the insertion, we check whether the current maximum right position is larger or equal than the current minimum left position, i.e., we compare the positions in the first entries of the two lists. If this test is positive, the free space intervals still have a non-empty intersection and we continue with adding the next horizontal cell boundary. If the test is negative or if the horizontal cell boundary did not contain free space, then there is no longer a non-empty intersection and we first set edge labels and modify the lists before we continue with the next horizontal cell boundary.

Let the $j$ th row be the first row for which the edge label has not been set and assume we have just added the horizontal cell boundary at row index $k<j$. If this cell boundary was empty we set all edge labels from $j$ to $k$ to $g, k$, where $g$ is the column index stored as third list entry in the last list elements of both lists. Then we delete all entries of both lists. If the cell boundary was not empty, then the minimum right position was smaller than the maximum left position. One of these two positions is in the just added row index $k$. Assume w.l.o.g. that the minimum right position is at row index $l$, with $k \leq l \leq j$, and the maximum left position is at row index $k$. Then we set the edge labels for rows $j$ to $l$ to $g, l$, where $g$ is the third list entry stored in the right list for row index $l$. Then we delete all entries of the right list up to and including $l$. We proceed by again comparing the top list elements. If now the minimum right position is not smaller than the maximum left position, i.e., there is a non-empty intersection, we can add the next horizontal cell 
boundary at row index $k-1$. Else, we also set the edge labels to the next entry of the right list, and so on.

Time and Space Complexity. In the update of $l_{t}$ we need to compute the new critical points of this column and their edge labels. This takes $O(n)$ time as described above. Furthermore, we also need to propagate critical points horizontally. That is, we need to propagate the $O(n)$ points of the new row to the at most $l$ rows to the left, and we need to propagate the $O(n l)$ points of previous rows to the new column. This, and therefore the update of $l_{t}$, takes $O(n l)$ time in total. In the update of $l_{s}$ we also need to delete critical points propagated from the column being deleted. These are again $O(n l)$ points.

The query is done as before only on a larger graph. The query time is $O(n \ell+m \ell)=O(n \ell)$ because the $m$ cluster curves now consist of at most $n \ell$ downward and $m \ell$ leftward directed edges.

The space complexity of the algorithm increases to $O\left(n \ell^{2}\right)$ since we now also store all horizontally propagated points. These are at most $\ell$ points per cell in at most $n \ell$ cells.

Theorem 3 A 2-distance approximation of the $\mathrm{SC}(m, \max , d)$ problem can be computed in $O\left(n^{2} \ell\right)$ time and $O\left(n \ell^{2}\right)$ space using the Fréchet distance in the case that all subtrajectories start and end at vertices of the trajectory where $n$ denotes the number of vertices of the trajectory and $\ell$ denotes the maximum number of vertices occurring on a reference trajectory in a cluster of $m$ curves.

\subsubsection{Reference Subtrajectory Starts and Ends at Vertices}

In this section, we consider the continuous Fréchet distance with the restriction that only the reference subtrajectory has to start and end at vertices and that each subtrajectory must contain at least one vertex of $T$. We can use the same algorithm as in the previous Section 4.2.1. The main modification we need is that we perform the query on all points and not only on the vertices of the free space. This does not change the time and space complexity of the algorithm.

The algorithm stays correct, because in fact a stronger version of Observation 1 holds. Namely, let $P$ be an arbitrary monotone curve in the free space from $v$ to $w$ where both $v, w$ are points on a vertical cell boundary. Then, by the definition of the propagated critical points, there will also be a monotone polygonal curve $P^{\prime}$ from the first critical point above $v$ to the first critical point below $w$ such that the vertices of $P^{\prime}$ are all vertices in $R$. Furthermore, the $y$-span of $P^{\prime}$ is a subset of the $y$-span of $P$.

We also need the following minor modification to the query operation. We now consider subtrajectories starting and ending at arbitrary points of $T$ and we have no restriction on the minimum length of a subtrajectory. Therefore a subtrajectory cluster may also consist of a set of points of $T$. In particular, a situation as described in Section 7 can occur. We can detect these situations as follows (and prevent our algorithm from running in an infinite loop). When we find a horizontal path in free space, i.e., a subtrajectory consisting of a single point, then we jump to the next critical point below. If from this point we again find a horizontal path, then we know by the convexity of free space cells, that there is a corridor of width larger than 0 in the free space. In this corridor, there are infinitely many horizontal paths, i.e., we have found infinitely many subtrajectories consisting of single points. In particular, the cluster has size at least $m$ and we can stop the query by returning true.

In total we have the following theorem.

Theorem 4 A 2-distance approximation of the $\mathrm{SC}(m, \max , d)$ problem can be computed in $O\left(n^{2} \ell\right)$ time and $O\left(n \ell^{2}\right)$ space using the Fréchet distance in the case when the reference subtrajectory must start and end at vertices of the trajectory where $n$ denotes the number of vertices of the trajectory 
and $\ell$ denotes the maximum number of vertices occurring on a reference trajectory in a cluster of $m$ curves.

\subsubsection{Arbitrary Subtrajectories}

We now consider the case where all subtrajectories (including the reference subtrajectory) may start and end at arbitrary points on the given trajectory. For this, we use the same algorithm as in Section 4.2.2 and extend it to handle the case of the reference subtrajectory not starting and ending at vertices of the trajectory.

The algorithm requires two major changes: (1) we add more critical points and event points and (2) we also need to search for optimal solutions in between event points. The new critical and event points are points where free space starts or ends within a cell. For the searching in between event points we need to do computation on the quadratic curves describing the cell boundaries of a free space cell. To our knowledge, this is the first algorithm explicitly working on the boundaries of free space cells.

First, we add more critical points to the free space, namely those points where - intuitively - free space starts or ends within a cell. That is, all points where the intersection of a vertical segment with the free space is a single point. See Figure 8 for an example. These critical points are also propagated to the right in the free space. The total number of critical points introduced in each cell is still at most eight. We will use these new critical points also as new event points, thus we now have $O\left(n^{2}\right)$ event points.

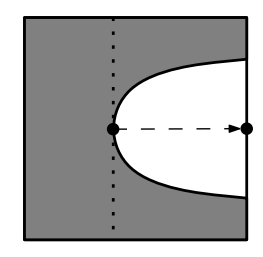

Figure 8: Further critical points needed for arbitrary subtrajectories.

With the added critical points and event points, we run the same algorithm as in the previous section. However, now in between event points we also need to find the longest cluster of $m$ curves. Suppose we have just moved either the sweep line $l_{s}$ to $l_{s+1}$ or $l_{t-1}$ to $l_{t}$. And suppose we have found $m$ cluster curves between $l_{s+1}$ and $l_{t-1}$ but not between $l_{s}$ and $l_{t-1}$ or $l_{s+1}$ and $l_{t}$, respectively. Then we search for the longest cluster of $m$ curves between $l_{s}$ and $l_{t}$. For this we first select a set of possible curves, which may have dependencies between each other. Figure 9 shows an example of a dependency: Suppose the sweep line $l_{s}$ is at the left vertical dashed line. If we want to include both curves in the cluster then $l_{t}$ may not be to the right of the right vertical dashed line. We can consider the largest feasible $t$ as a function in $s$. This is an algebraic function of degree at most 4 since the free space boundaries are algebraic functions of degree at most 2 [4].

We can select a set of at most $n$ possible curves, in which each curve may have a dependency on at most two other curves (to one curve above and one curve below). For this, we start by finding and selecting the top most curve as before. Dependencies only occur between a curve ending at the top most point of an interval and a curve starting at the bottom most point of an interval (see again Figure 9). Thus, when a selected curve ends on a top most point, we also add - if this exists - the dependent curve starting at the bottom most but higher point on $l_{t}$ in the same row. Furthermore, we add as before the next non-dependent curve starting at the same height or lower. Because dependent curves start on bottom most points, the dependent and non-dependent curve start in different rows. Thus, we get at most one curve starting in each row, and $n$ curves in total. 


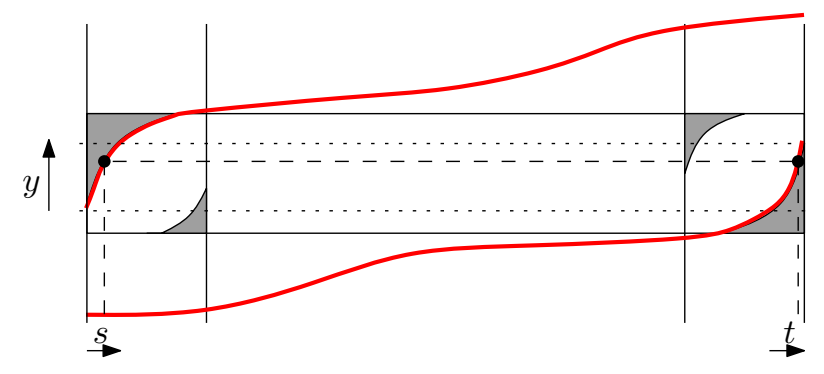

Figure 9: A dependency between two cluster curves for arbitrary subtrajectories. The dotted lines indicate the region of overlap in the $y$-coordinate.

We can have at most $n_{d}<n$ such dependencies since we have at most one dependency per row of the free space diagram.

In total, the set of possible curves contains at most $n$ dependencies (at most one per row). For these dependencies, we consider the arrangement of the functions $\ell_{i}(s):=t_{i}(s)-s$ where $t_{i}(s)$ is the largest feasible $t$ for $s$ for the $i$ th dependency $(1 \leq i \leq 2 n)$. If we have $m$ compatible curves in the free space at a position in the arrangement then we still have these curves at any position in the corresponding cell of the arrangement. We therefore only need to consider vertices of the arrangement and local maxima of the functions $\ell_{i}$.

We do not need to consider all levels of the arrangement. Suppose we are below the $(m-1)$ st level. Furthermore, we do not need to consider the whole arrangement but only the $\leq(m-1)$ level, where we count levels from the top, e.g., the 0th level is the upper envelope. Below the $(m-1)$ st level we have $m-1$ pairs of compatible curves. This yields at least $m$ compatible curves, for instance by choosing the top most curve, and from each pair of curves the lower curve. The $\leq(m-1)$-level has complexity $O\left(m^{2} \lambda_{4}(n / m)\right)=O\left(n m 2^{\alpha(n / m)}\right)$ and can be computed in $O\left(n m 2^{\alpha(n / m)} \log n \log (n / m)\right)$ time using a simple divide and conquer algorithm [39] (see [1] for incremental algorithms). The space needed for the computation is in $O\left(n m 2^{\alpha(n / m)}\right)$. We need to assume that the model of computation allows to compute the intersections of two of these functions in constant time. To compute local maxima we further need to assume that the model of computation allows to compute these in constant time.

This gives us $O\left(n m 2^{\alpha(n / m)}\right)$ vertices of the arrangement and local maxima of the functions at which the maximum length might be achieved. For these points we can query whether there are $m$ curves in $O(m)$ time. For this, we store the $n$ possible curves in an array with pointers from each curve to the next dependent and non-dependent curve.

It remains to check for horizontal paths starting between $l_{s}$ and $l_{s+1}$ and ending between $l_{t-1}$ and $l_{t}$. For such a path the end point might depend on the start point but we can find the longest horizontal path by solving the corresponding algebraic equation. If there is a horizontal corridor, i.e., an infinite number of path close to the longest path, then this yields a cluster of length arbitrary close to the longest horizontal path.

In total there are $O\left(n^{2}\right)$ events and for each event we build the arrangement for the $\leq(m-1)$ levels which requires $O\left(n m 2^{\alpha(n / m)} \log n \log (n / m)\right)$ time. Each of the $O\left(n m 2^{\alpha(n / m)}\right)$ vertices in the arrangement can be checked in $O(m)$ time. Thus, we have obtained the following results for subtrajectory clustering under the continuous Fréchet distance.

Theorem 5 A 2-distance approximation of the $\mathrm{SC}(m, \max , d)$ problem can be computed in time $O\left(n^{3} m 2^{\alpha(n / m)}(\log n \log (n / m)+m)\right)$ using $O\left(n \ell^{2}+n m 2^{\alpha(n / m)}\right)$ space using the continuous Fréchet distance for arbitrary subtrajectories where $n$ denotes the number of vertices of the trajectory and $\ell$ denotes the maximum number of vertices occurring on a reference trajectory in a cluster of $m$ 
curves. In these bounds, $n$ denotes the size of the given trajectory and $\ell$ the length of the longest subtrajectory cluster found, and we assume that we can find the local maxima and intersections of algebraic functions of degree 4 in constant time.

Instead of querying for all of the points we can perform a binary search on the levels, i.e., on the range 0 to $m-1$. By this we query for at most $O\left(\min \left\{n m 2^{\alpha(n / m)}, \log m L(n, m)\right\}\right)$ points, where $L(n, m)$ denotes the worst case complexity of the $m$ th level. While we assume that the binary search improves the worst-case run-time, from the known upper bounds on $L(n, m)$ we only get an improvement if $m$ is large. For instance Chan [14] proved that $L(n, m) \in O\left(n^{2-1 / 6-\delta}\right)$ for a positive constant $\delta$.

\section{Extensions}

The algorithms described above can be modified to handle various other settings. Here we describe how to deal with clustering subtrajectories of a set of trajectories rather than one big one, how to optimise the size of the cluster rather than the length, and how to use a different distance metric: the weak Fréchet distance.

\subsection{Subtrajectory Cluster of a Set of Trajectories}

The above algorithms can easily be extended to handle the case when the input is a set $S$ of trajectories and the aim is to find the longest subtrajectory cluster of $S$, allowing either one or several subtrajectories of each trajectory in $S$. First we consider the case where several subtrajectories of one trajectory are allowed. Two simple modifications are needed to handle these cases. Join all trajectories into one long trajectory $T$ of length $k n$, and build the free space diagram $\mathcal{F}_{d}(T, T)$. As before we sweep two vertical lines $l_{s}$ and $l_{t}$ from left to right. However, we only consider the cases when $l_{s}$ and $l_{t}$ lie on the same trajectory in $S$. Furthermore, when checking the number of cluster curves between $l_{s}$ and $l_{t}$ in the free space diagram we only consider cluster curves that start and end on the same trajectory (their $y$-coordinates is included in the interval spanned by one trajectory). These changes are trivial and do not change the complexity bounds of the algorithm. For $k$ trajectories of complexity $n_{1}, \ldots, n_{k}$, where $\sum_{i=1}^{k} n_{i}=n$, the joint freespace diagram has size $n \times n$.

Next we consider the case where only one subtrajectory of each trajectory is allowed. We could check this during the query operation. But in fact, for this variant of the problem a simpler data structure suffices. Namely, for each vertex of the data structure it suffices to store the starting index of the longest path. Thus, each point on $l_{t}$ requires only constant memory and we do not need to store any further information. This reduces the space complexity to $O(n)$ using the discrete Fréchet distance and $O(\ell n)$ using the continuous Fréchet distance if all or only the reference trajectory starts and ends at vertices. The query can be done in $O(k)$ time by storing for each trajectory whether it currently contains a similar subtrajectory. This value is updated in the update of $l_{t}$ by checking if any points on the trajectory store $l_{s}$ as index. However, the time for updating $l_{t}$ and therefore for the continuous Fréchet distance the total asymptotic running time stay the same.

Finally, we consider the case of the continuous Fréchet distance with arbitrary subtrajectories. In this case we again get additional event points which not change the number of event points asymptotically. For simplicity we allow only subtrajectories containing at least a vertex. Otherwise the subtrajectories from the different trajectories could be single points that depend on each other. These trajectories can be resolved as the dependencies in Section 4.2.3. Without these dependencies the asymptotic running time for arbitrary subtrajectories is as in the case when either all or only the reference subtrajectory must start and end at a vertex. 
Theorem 6 A 2-distance approximation of the longest subtrajectory cluster in a given set of trajectories problem of $k$ trajectories of total complexity $n$, where one trajectory may be chosen, can be computed in

- $O\left(n^{2}\right)$ time and $O(n)$ space using the discrete Fréchet distance,

- $O\left(\ell n^{2}\right)$ time and $O(\ell n)$ space using the continuous Fréchet distance where either all or only the reference subtrajectory must start and end at vertices of the trajectory,

- $O\left(\ell n^{2}\right)$ time and $O(\ell n)$ space using the continuous Fréchet distance for arbitrary subtrajectories if each subtrajectory must contain at least one vertex,

where $n$ denotes the total number of vertices of the trajectories and $\ell$ denotes the maximum number of vertices occurring on a reference trajectory in a cluster of $m$ curves. If more than one subtrajectory of each trajectory is allowed, the complexities are the same as for one trajectory and several subtrajectories.

\subsection{Maximising the Number of Cluster Curves}

The algorithm can easily be modified to solve the 2-distance approximation of the $\mathrm{SC}(\max , \ell, d)$ problem. For the discrete Fréchet distance and the continuous Fréchet distance with restricted vertices we report a maximum cluster with length at least $\ell$. For this optimisation version, the algorithm slides the sweep lines $l_{s}$ and $l_{t}$ with distance $\ell$ over the free space diagram. Note that the distance $\ell$ is measured along the reference subtrajectory and not in the free space. (Alternatively, we can scale the free space such that the lengths coincide.)

For the case where subtrajectories start and end at vertices, the algorithm proceeds as follows. In the first step, the line $l_{t}$ is swept from position 0 to position $\ell$. In all subsequent steps, we move first $l_{s}$ one index and then $l_{t}$ to the first index such that the distance is at least $\ell$. These are a linear number of positions in total. At each position, the query returns the number of cluster curves and the maximum of these numbers is returned.

For the case of arbitrary subtrajectories we do not need to compute the arrangement of curves corresponding to the dependencies between curves. Instead we can restrict us to the values of $s$ where $\ell_{i}(s)=\ell$ for the $i$ th dependency $(1 \leq i \leq 2 n)$.

Theorem 7 A 2-distance approximation of the $\mathrm{SC}(\max , \ell, d)$ problem can be computed in

- $O\left(n^{2}+\ell m n\right)$ time and $O(\ell n)$ space using the discrete Fréchet distance,

- $O\left(\ell n^{2}\right)$ time and $O\left(\ell^{2} n\right)$ space using the continuous Fréchet distance where either all or only the reference subtrajectory must start and end at vertices of the trajectory,

- $O\left(m n^{3}\right)$ time and $O\left(\ell^{2} n\right)$ time and space using the continuous Fréchet distance for arbitrary subtrajectories,

where $n$ denotes the number of vertices of the trajectory and $m$ denotes the maximum number of subtrajectories in a cluster of length at least $\ell$. 


\subsection{Weak Fréchet Distance}

The weak Fréchet distance is defined as the Fréchet distance except that the functions (in Definitions 2 and 3) do not need to be increasing. For the weak discrete Fréchet distance in the couplings (in Definition 4) the indices may also decrease by 1 .

We use a similar algorithm as for the discrete and continuous Fréchet distance. However, now the cluster curves do not need to be $x$ - and $y$-monotone. We store the free space as an undirected, unlabelled graph. For the continuous Fréchet distance instead of the vertical propagation that we used in Section 4.2.1, we now simply add one vertex for each horizontal cell boundary. The space complexity in all cases is simple $O(\ell n)$ (plus the cost of storing the arrangement in the case of the continuous Fréchet distance for arbitrary subtrajectories.

For the query, we again greedily search for topmost paths. However, now we may use both edge directions and cannot prevent running into dead ends. In this case we backtrack and need to remember which edges we have already taken. The query can be done greedily: find the topmost path from $l_{t}$ to $l_{s}$ in the graph and continue with the graph but now no vertex is allowed to be higher than the lowest vertex of the previous path. This query takes $O(\ell n)$ time since we have to search at most the whole graph.

Theorem 8 A 2-distance approximation of the $\mathrm{SC}(m, \max , d)$ problem can be computed in

- $O\left(\ell n^{2}\right)$ time and $O(\ell n)$ space using the weak discrete Fréchet distance,

- $O\left(\ell n^{2}\right)$ time and $O(\ell n)$ space using the weak continuous Fréchet distance where either all or only the reference subtrajectory must start and end at vertices of the trajectory,

- $O\left(n^{3} m 2^{\alpha(n / m)}(\log n \log (n / m)+m)\right)$ time and $O\left(\ell n+n m 2^{\alpha(n / m)}\right)$ space using the weak continuous Fréchet distance for arbitrary subtrajectories,

where $n$ denotes the number of vertices of the trajectory and $\ell$ denotes the maximum number of vertices occurring on a reference trajectory in a cluster of $m$ curves.

\section{A Note on the Experiments}

To test our ideas we implemented two simplified versions of the algorithm presented in Section 4.2. Both implementations build upon an earlier version of our algorithm which had a running time of $O\left(n^{2} \ell^{2}\right)$. The first (naïve) approach precomputes the entire free space and hence uses $O\left(n^{2}\right)$ space, the second algorithm builds the free space during the sweep and uses only $O(n \ell)$ space. The experiments were performed on an Intel Pentium-4 3.0 GHz processor and 1GB of RAM. The clustering algorithms were implemented and compiled using Java SE Development Kit 6 under the Eclipse SDK environment. The test data was generated using a modified version of NetLogo [45] and generated a set of trajectories whose complexity varied from 50 to 25,000. We measured the time required to run different experiments as well as the amount of memory each experiment consumed. Each experiment was performed three times and the number in the table is the average value of the running times.

As can be seen in the table the running time increases rapidly with the complexity of the trajectory. To test the usefulness of our algorithm we tested it on a real trajectory. The trajectory was obtained by carrying a GPS for one month in a $50 \times 50 \mathrm{~km}$ region and it generated 82,160 time-points. Running the memory efficient algorithm without any optimisation options took approximately 7 hours and used $42 \mathrm{MB}$ of memory. This is not manageable in practice but there are many simple ideas that can be used to improve the running time. A simple and effective improvement is to simplify the trajectory [26]. Using a distance threshold of 10 meters compressed the 
Table 1: Result of clustering algorithm given input of a trajectory

\begin{tabular}{|r||r|r||r|r|}
\hline \multicolumn{1}{|c||}{ Total Points } & \multicolumn{2}{c||}{ Standard Mode } & \multicolumn{2}{c|}{ Memory Efficient Mode } \\
\hline & Time (sec) & Memory (MB) & Time (sec) & Memory (MB) \\
\hline \hline 50 & 0.07 & 1.14 & 0.03 & 0.67 \\
\hline 75 & 0.08 & 2.59 & 0.08 & 0.70 \\
\hline 250 & 0.25 & 7.51 & 0.20 & 0.89 \\
\hline 375 & 0.44 & 18.93 & 0.28 & 1.31 \\
\hline 500 & 0.81 & 27.78 & 0.62 & 1.34 \\
\hline 1000 & 3.59 & 120.53 & 1.66 & 5.66 \\
\hline 2500 & \multicolumn{2}{|c||}{ N/A } & 13.71 & 7.30 \\
\hline 5000 & \multicolumn{2}{||}{ N/A } & 88.28 & 7.32 \\
\hline 7500 & \multicolumn{2}{|c|}{ N/A } & 2227.08 & 9.15 \\
\hline 25000 & \multicolumn{2}{|c|}{ N/A } & & 10.73 \\
\hline
\end{tabular}

trajectory to 5,228 time-steps and is done in about $170 \mathrm{~ms}$. With the compressed trajectory the memory efficient algorithm computed the clusters in roughly 2 minutes using $2.65 \mathrm{MB}$ of memory where the distance threshold $d$ was set to 15 meters. Fig. 10 shows the trajectory. The parts in the longest cluster are thickened and shown in red.

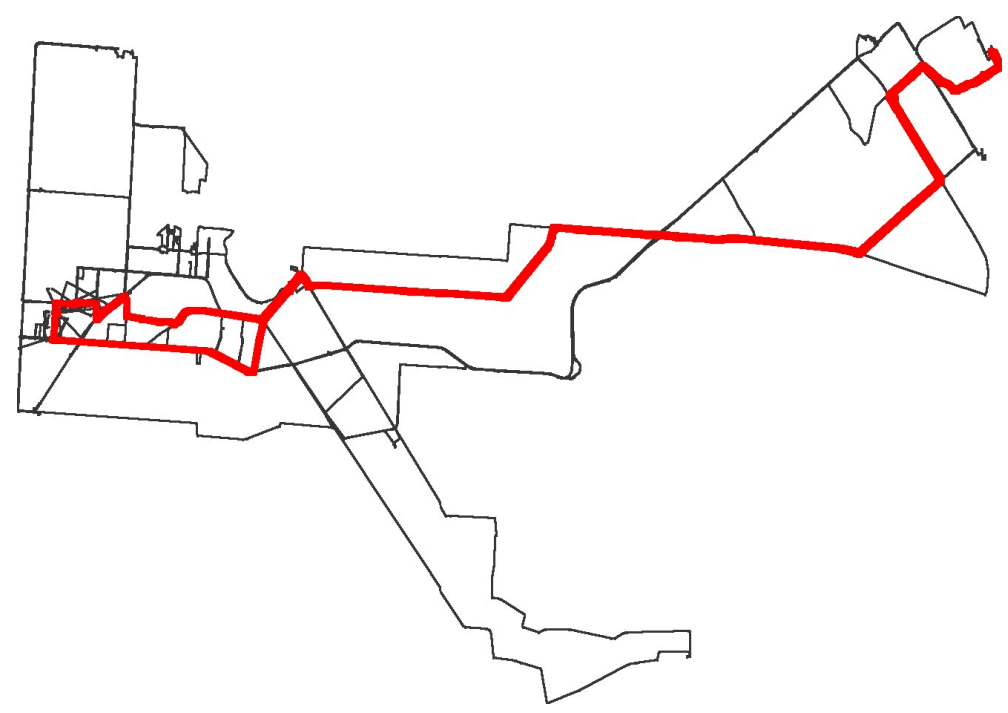

Figure 10: A trajectory with 5,228 time-points where the longest trajectory cluster is marked.

The approach looks promising and we believe the running time can be improved considerably by simple ideas, such as pruning the free space so that time intervals that are obviously not of any interest are omitted with only minor preprocessing.

\section{Conclusion}

Our definition of the length of a cluster measures the length of the longest curve in a cluster. Theoretically, this allows the case of a cluster consisting of one curve of length $\ell$ and (in the case of arbitrary subtrajectories) infinitely many subtrajectories of length 0, i.e., points. However, this can occur only when the input trajectory has a subtrajectory of length $\ell$ that stays inside a disk of 
radius $d$. In practice, this is unlikely to occur. To eliminate this problem, an alternative approach could be to measure and optimise the length of the shortest curve in the cluster. However, this seems much harder to handle algorithmically. Currently in the sweep we aim at cluster curves with a short $y$-span (and long $x$-span). For maximising the length of the shortest curve we would need to find cluster curves with large $x$ - and $y$-spans.

\section{Acknowledgement}

The authors would like to thank Matthew Lowry for introducing us to the problem and giving several insightful comments and Cahya Ong for the implementation.

\section{References}

[1] P. K. Agarwal, M. de Berg, J. Matousek, and O. Schwarzkopf. Constructing Levels in Arrangements and Higher Order Voronoi Diagrams. Proc. 10th Annual ACM Symposiom on Computational Geometry, pp. 67-75, 1994.

[2] R. Agrawal, K.-I. Lin, H. S. Sawhney, and K. Shim. Fast similarity search in the presence of noise, scaling, and translation in time-series databases. Proc. 21st International Conference on Very Large Data Bases, pp. 490501, 1995.

[3] G. Al-Naymat, S. Chawla and J. Gudmundsson. Dimensionality Reduction for Long Duration and Complex Spatio-Temporal Queries. Proc. 22nd ACM Symposium on Applied Computing, pp. 393-397, 2007.

[4] H. Alt and M. Godau. Computing the Fréchet distance between two polygonal curves. International Journal on Computational Geometry and Applications, 5:75-91, 1995.

[5] H. Alt and L. Guibas. Discrete geometric shapes: Matching, interpolation, and approximation. In J.-R. Sack and J. Urrutia, editors, Handbook of Computational Geometry, pp. 121-153. Elsevier, 1999.

[6] H. Alt, C. Knauer and C. Wenk. Comparison of Distance Measures for Planar Curves. Algorithmica, 38(2):45-58, 2004.

[7] M. Andersson, J. Gudmundsson, P. Laube and T. Wolle. Reporting Leadership patterns Among Trajectories. GeoInformatica, 12(4):497-528, 2008.

[8] M. Benkert, B. Djordjevic, J. Gudmundsson and T. Wolle. Finding popular places. Proc. 18th International Symposium on Algorithms and Computation, 2007.

[9] M. Benkert, J. Gudmundsson, F. Hübner and T. Wolle. Reporting Flock Patterns. Computational Geometry : Theory and Applications, 41(3):11-125, 2008.

[10] K. Buchin, M. Buchin and J. Gudmundsson. Detecting Single File Movement. To appear in Proc. 16th ACM SIGSPATIAL International Conference on Advances in Geographic Information Systems (ACM GIS), 2008.

[11] K. Buchin, M. Buchin, C. Knauer, G. Rote and C. Wenk. How Difficult is it to Walk the Dog? In Proc. 23rd European Workshop on Computational Geometry, pp. 170-173, 2007.

[12] K. Buchin, M. Buchin and Y. Wang. Exact Algorithm for Partial Curve Matching via the Fréchet Distance. To appear in Proc. 20th ACM-SIAM Symposium on Discrete Algorithms, 2009 . 
[13] H. Cao, O. Wolfson and G. Trajcevski. Spatio-temporal data reduction with deterministic error bounds. The VLDB Journal, 15(3):211-228, 2006.

[14] T. Chan. On levels in arrangements of curves, III: further improvements. In Proc. 24th ACM Symposium on Computational Geometry (SoCG), pp. 85-93, 2008.

[15] A. F. Cook IV and C. Wenk. Geodesic Fréchet Distance Inside a Simple Polygon. In Proc. 25th Annual Symposium on Theoretical Aspects of Computer Science (STACS), pp. 193-204, 2008.

[16] A. Dumitrescu and G. Rote. On the Fréchet distance of a set of curves. Proc. 16th Canadian Conference on Computational Geometry, pp. 162-165,2004.

[17] T. Eiter and H. Mannila. Computing discrete Fréchet distance. Tech. Report CD-TR 94/64, Information Systems Department, Technical University of Vienna, 1994.

[18] J. Erickson and R. Seidel. Better Lower Bounds on Detecting Affine and Spherical Degeneracies. Discrete \& Computational Geometry 13:41-57, 1995.

[19] A. U. Frank. Socio-Economic Units: Their Life and Motion. In A. U. Frank, J. Raper, and J. P. Cheylan, editors, Life and motion of socio-economic units, volume 8 of GISDATA, pp. 21-34. Taylor \& Francis, London, 2001.

[20] S. Gaffney and P. Smyth. Trajectory Clustering with Mixtures of Regression Models. In Proc. 5th ACM SIGKDD International Conference on Knowledge Discovery and Data Mining, pp. 63-72, 1999.

[21] S. Gaffney, A. Robertson, P. Smyth, S. Camargo and M. Ghil. Probabilistic Clustering of Extratropical Cyclones Using Regression Mixture Models. Climate Dynamics, 29(4), pp. 423440, 2007.

[22] A. Gajentaan and M. H. Overmars. On a Class of $O\left(n^{2}\right)$ Problems in Computational Geometry. Computational Geometry - theory and applications, 5:165-185, 1995.

[23] M. R. Garey and D. S. Johnson. Computers and intractability. W. H. Freeman, 1979.

[24] J. Gudmundsson and M. J. van Kreveld. Computing longest duration flocks in trajectory data. Proc. 14th ACM Symposium on Geographic Information Systems, pp. 35-42, 2006.

[25] J. Gudmundsson, P. Laube, and T. Wolle. Encyclopedia of GIS, chapter Movement Patterns in Spatio-Temporal Data. Springer, 2008. To appear.

[26] J. Gudmundsson, J. Katajainen, D. Merrick, C. Ong and T. Wolle. Compressing spatiotemporal trajectories. Proc. 18th International Symposium on Algorithms and Computation, 2007.

[27] J. Gudmundsson, M. van Kreveld and B. Speckmann. Efficient detection of motion patterns in spatio-temporal data sets. GeoInformatica, 11(2):195-215, 2007.

[28] U. I. Gupta, D. T. Lee and J. Y.-T. Leung. Efficient algorithms for interval graphs and circular-arc graphs. Networks, 12:459-467, 1982.

[29] R. H. Güting and M. Schneider. Moving Objects Databases. Morgan Kaufmann Publishers, 2005 .

[30] M. Hadjieleftheriou, G. Kollios, V. J. Tsotras and D. Gunopulos. Indexing spatio-temporal archives. The VLDB Journal, 15(2):143164, 2006.

[31] J. Håstad. Clique is hard to approximate within $n^{1-\varepsilon}$. ACTA Mathematica, 182:105-142, 1999. 
[32] P. Kalnis, N. Mamoulis and S. Bakiras. On discovering moving clusters in spatio-temporal data. Proc. 9th International Symposium on Advances in Spatial and Temporal Databases, volume 3633 of LNCS, pp. 364-381. Springer, 2005.

[33] K. Kalpakis, D. Gada and V. Puttagunta. Distance measures for effective clustering of arima timeseries. Proc. 1st IEEE International Conference on Data Mining pp. 273280, 2001.

[34] P. Laube, S. Imfeld and R. Weibel. Discovering relative motion patterns in groups of moving point objects. International Journal of Geographical Information Science, 19(6):639-668, 2005 .

[35] P. Laube, M. van Kreveld and S. Imfeld. Finding REMO - detecting relative motion patterns in geospatial lifelines. Developments in Spatial Data Handling: Proceedings of the 11th International Symposium on Spatial Data Handling, pp. 201-214, 2004.

[36] N. Mamoulis, H. Cao, G. Kollios, M. Hadjieleftheriou, Y. Tao, and D. Cheung. Mining, indexing, and querying historical spatiotemporal data. Proc. 10th ACM SIGKDD International Conference On Knowledge Discovery and Data Mining, pp. 236-245, 2004.

[37] M. Nanni and D. Pedreschi. Time-focused density-based clustering of trajectories of moving objects. Journal of Intelligent Information Systems, 27(3):267-289, 2006.

[38] J.-G. Lee, J. Han and K.-Y. Whang. Trajectory clustering: a partition-and-group framework. Proc. ACM SIGMOD International Conference on Management of Data, pp. 593-604, 2007.

[39] M. Sharir. On $k$-sets in arrangements of curves and surfaces. Discrete \& Computational Geometry, 6:593-613, 1991.

[40] S. Sâltenis, C. S. Jensen, S. T. Leutenegger and M. A. Lopez. Indexing the positions of continuously moving objects. Proc. ACM SIGMOD International Conference on Management of Data, pp. 331-342, 2000.

[41] R. C. Veltkamp and M. Hagedoorn. State-of-the-art in shape matching. Principles of Visual Information Retrieval, M. Lew (ed.), pp. 87-119, Springer, 2001.

[42] F. Verhein and S. Chawla. Mining spatio-temporal association rules, sources, sinks, stationary regions and thoroughfares in object mobility databases. Proc. 11th International Conference on Database Systems for Advanced Applications (DASFAA), volume 3882 of LNCS, pp. 187201. Springer, 2006.

[43] M. Vlachos, D. Gunopulos and G. Kollios. Discovering Similar Multidimensional Trajectories. Proc. 18th International Conference on Data Engineering, pp. 673-684, 2002.

[44] Wildlife tracking projects with GPS GSM collars. www.environmental-studies.de/projects/projects.html, 2007.

[45] U. Wilensky. NetLogo (and NetLogo User Manual). http://ccl.northwestern. edu/netlogo, 1999. 